\title{
Corrigendum
}

\section{CTLA4 aptamer delivers STAT3 siRNA to tumor-associated and malignant $\mathrm{T}$ cells}

Andreas Herrmann, ${ }^{1}$ Saul J. Priceman, ${ }^{1}$ Piotr Swiderski, ${ }^{2}$ Maciej Kujawski, ${ }^{1}$ Hong Xin, ${ }^{1}$ Gregory A. Cherryholmes, ${ }^{1}$ Wang Zhang, ${ }^{1}$ Chunyan Zhang, ${ }^{1}$ Christoph Lahtz, ${ }^{1}$ Claudia Kowolik, ${ }^{3}$ Steve J. Forman, ${ }^{4}$ Marcin Kortylewski, ${ }^{1}$ and $\mathrm{Hua} \mathrm{Yu}^{1}$

${ }^{1}$ Department of Cancer Immunotherapeutics and Tumor Immunology, ${ }^{2}$ Biopolymer Core, ${ }^{3}$ Department of Molecular Medicine, and ${ }^{4}$ Department of Hematology and Hematopoietic Cell Transplantation, Beckman Research Institute at City of Hope, Comprehensive Cancer Center, Duarte, California, USA.

Original citation: J Clin Invest. 2014;124(7):2977-2987. doi:10.1172/JCI73174.

Citation for this corrigendum: J Clin Invest. 2015;125(6):2547. doi:10.1172/JCI82555.

In the original manuscript, Piotr Swiderski was omitted from the author list, and Steve J. Forman's affiliation was incorrect. The correct author and affiliation lists are above. Supplemental Figure 1A included an incorrect sequence for the CTLA4-targeting aptamer and omitted the presence of an additional linker region. The figure and legend have been corrected and updated online to include additional information about the design of the CTLA4-targeting aptamer. A sentence regarding the Biopolymer Core was omitted from the Acknowledgments. The correct sentence is below.

We thank the staff of the Biopolymer Core at the Beckman Research Institute for designing and synthesizing the CTLA4 4 apt_STAT3 siRNA conjugates.

The authors regret the errors. 